\title{
Nearly a Decade of Cosmic Ray Observations in the Very Local Interstellar Medium
}

\author{
Jamie Sue Rankin ${ }^{a}$, \\ ${ }^{a}$ Department of Astrophysical Sciences, Princeton University, Princeton, NJ 08544 \\ E-mail: jsrankineprinceton.edu
}

In 2012, the centennial year of the discovery of cosmic rays, Voyager 1 crossed the heliopause and began making the very first in-situ measurements of the surrounding interstellar medium. Joined by Voyager 2 in 2018, these twin spacecraft continue to provide critical data of cosmic rays in a surprising, previouslyunexplored plasma regime. Here, we highlight some of the discoveries and insights that have emerged from nearly a decade of cosmic ray observations in the very local interstellar medium, addressing topics such as: i) the behavior of cosmic rays at the heliopause boundary, ii) the characteristics of the low-energy spectrum (down to a few $\mathrm{MeV} / \mathrm{nuc}$ ), iii) the discovery of a time-varying, species-dependent anisotropy, and iv) their relationship to solar-transients that pass through the heliosphere and transmit pressure waves into the VLISM.

37th International Cosmic Ray Conference (ICRC 2021)

July 12th-23rd, 2021

Online-Berlin, Germany

\section{*Presenter}




\section{Introduction}

On August 25, 2012, at a radial distance of 121.6 au from the Sun, Voyager 1 became the first spacecraft to exit the heliosphere and directly sample the Very Local Interstellar Medium ${ }^{1}$. The signatures of its crossings were at first, ambiguous to interpret. The energetic particle response was characterized by a sudden increase in cosmic ray intensity that coincided with a sudden depletion of solar particles (Figure 1) $[1,2,3]$, indicating a clear transition into a new region. The nature and magnitude of the magnetic field also changed from the weak, turbulent field of the heliosheath $(0.13 \mathrm{nT})$ to that of the stronger, smoother field expected for the interstellar medium $(0.49 \mathrm{nT})[4,5]$, but its direction remained consistent with that of the Parker Spiral in the heliosphere. An absence of direct plasma measurements caused Voyager 1 to probe the environment using other means. Fortuitous radio emissions resulting from electron plasma oscillations were eventually used to confirm interstellar density values; these revealed plasma densities $\left(0.08 \mathrm{~cm}^{-3}\right)$ that were more consistent with estimations for the VLISM $\left(0.1 \mathrm{~cm}^{-3}\right.$ expected) than the heliosheath $\left(\sim 0.002 \mathrm{~cm}^{-3}\right)[6,7]$. These collective findings revealed that Voyager 1 had begun a new phase of its journey - one of interstellar exploration. On November 5, 2018, Voyager 2 joined its twin, and, from contrasting vantage points, the two spacecraft continue to explore this new region surrounding the heliopause.

Despite the significant differences in the spacecraft trajectories, ${ }^{2}$ the heliopause crossings occurred at comparable radial distances (121.6 vs. $119.0 \mathrm{au}$ ) and were marked by similarly abrupt transitions in the energetic particles and magnetic fields (under a day), from which [5] calculated a heliopause thickness of $<5 \times 10^{-3}$ au (assuming it was stationary). The plasma wave instruments onboard both spacecraft (PWS) showed a clear ramp in plasma density: from $\sim 0.002 \mathrm{~cm}^{-3}$ in the heliosheath to $0.055 \mathrm{~cm}^{-3} \& 0.039 \mathrm{~cm}^{-3}$ in the VLISM (for Voyagers $1 \& 2$, respectively, shortly after their crossings) - indicative of a plasma boundary layer roughly 10 au or more thick [7]. The magnetic field directions by both spacecraft also remained consistent with those in the heliosheath, a detail that is now captured in several models and generally attributed to the draping of the interstellar magnetic field around the heliosphere [5] (and references therein). Other details about the plasma were also finally obtained by the plasma instrument on Voyager 2 (PLS) [8]. For instance, prior to crossing it detected a plasma barrier 1.5 au thick with plasma twice as dense as typical heliosheath material and a thinner boundary layer ( 0.06 au before the heliopause), in which the density and magnetic field increased and the radial flow speed decreased. Data from PLS and PWS also revealed a variable VLISM that was hotter than expected (30,000 to 50,000 K compared to the predicted 15,000 to $30,000 \mathrm{~K}$ ).

\footnotetext{
${ }^{1}$ A re-defining of the term, as proposed by [77]: "that region of the interstellar medium surrounding the Sun that is modified or mediated by heliospheric processes or material."

${ }^{2}$ The location of Voyager 1's crossing occurred at 121.6 au, $255.0^{\circ}$, and $35.0^{\circ}$ (radial distance, longitude, and latitude), while Voyager 2's occurred at $119.0 \mathrm{au}, 290.3^{\circ}$, and $-32.2^{\circ}$ (solar ecliptic coordinates) [5].
} 
The two crossings also featured some noticeable differences, particularly in cosmic rays, as shown in Figure 1. For example, [9] reported that the upstream solar ions at Voyager 1 lasted for $\sim 25$ days ( $\sim 0.25 \mathrm{au})$ and gradually depleted through a generally-smooth reduction, while those at Voyager 2 lasted $\sim 65$ days $(\sim 0.6 \mathrm{au})$ and had a profile marked with substantial structure. [4] detailed how Voyager 1 encountered the boundary 5 times leading up to its final crossing, distinguished by jumps in the field that also coincided with reduction of low-energy particles. These phenomena now understood to result from flux interchange instability $[10,11]$ enabled magnetic flux tubes at the heliopause to connect to the outer heliosheath and provide a path for the particles to escape. Voyager 2's crossing, on the other hand, was preceded by a large magnetic barrier, a region of elevated magnetic field strength $(\sim 0.3 \mathrm{nT})$ and smoothly increasing cosmic ray
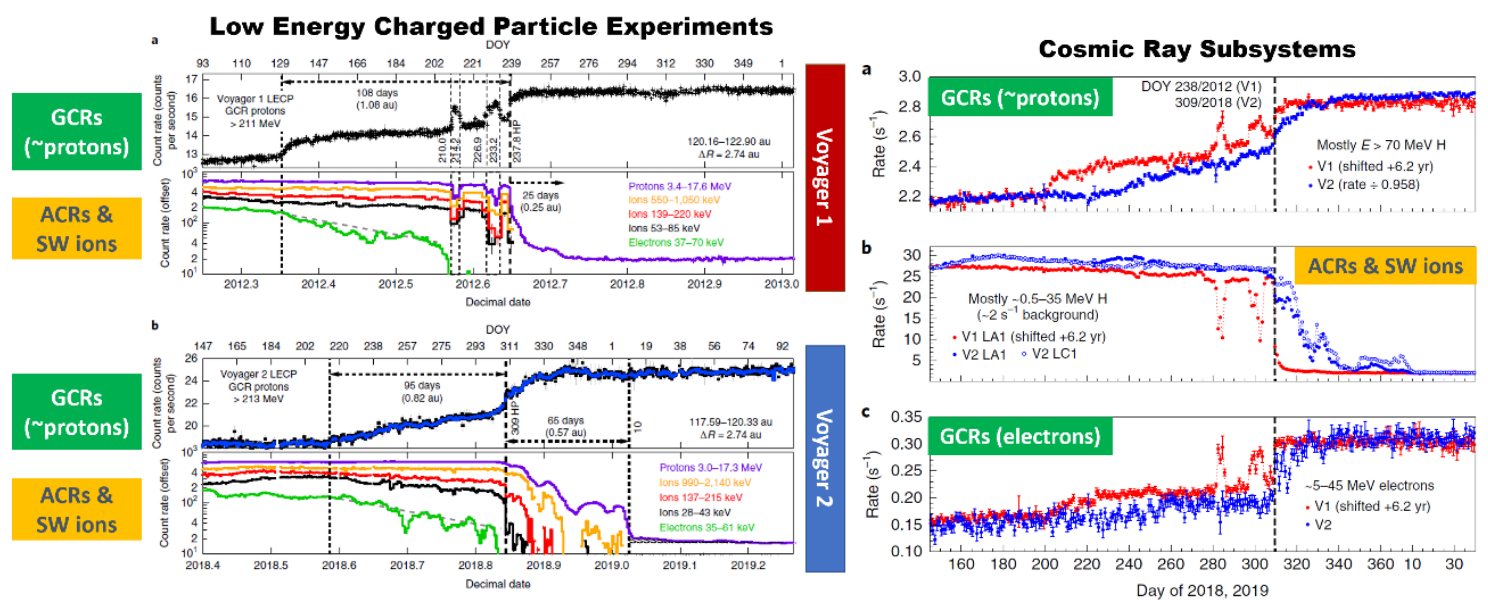

Figure 1. Cosmic ray count rates observed by the Low Energy Charged Particle experiments (LECP; Left) and Cosmic Ray Subsystems (CRS; Right) at each heliopause crossing. Panels showing galactic cosmic rays (GCRs) are noted in green, while those showing solar particles (ACRs \& SW ions) are noted in yellow. The two spacecrafts' measurements are differentiated by panel location in the left image (Voyager 1 on top and Voyager 2 on bottom), and by color in the right image (Voyager 1 in red and Voyager 2 in blue); adapted from $[9,26]$.

intensities that lasted $\sim 80$ days $(\sim 0.7 \mathrm{au})$. Oddly, the plasma in this region showed no corresponding change in the density, temperature or speed $[5,8]$.

The nature of the recently encountered VLISM juxtaposes that of the well-studied solar wind in many ways. The quiescence of the field and plasma, as well as the absence of solar modulation makes this an ideal environment for examining low-energy cosmic rays, particularly before their transport undergoes significant modification on their inward journey towards the Sun. Here, we review some of the discoveries and insights that have so far emerged from measuring cosmic rays in this new regime by highlighting several key observations: i) cosmic ray behavior at the heliopause boundary, ii) characteristics of the low-energy spectrum (down to a few $\mathrm{MeV} / \mathrm{nuc}$ ), iii) the discovery of a time-varying, species-dependent anisotropy, and iv) cosmic-ray disturbances caused by solar transients that pass through the heliosphere and transmit pressure waves into the VLISM.

\section{Cosmic Rays at the Heliopause Boundary}

The abruptness of the cosmic ray transition at the heliopause crossing came as somewhat of a surprise and hints at an interesting contrast between the heliosheath and VLISM plasmas. Although some change in the diffusion coefficient and cosmic ray intensities was expected, several models predicted a much more gradual transition than what was observed. For example, [12] argued that $100 \mathrm{MeV}$ protons beyond the heliopause should increase by $25 \%$ to $40 \%$ over a distance of $100 \mathrm{au}$, implying an average gradient of $0.25 \% \mathrm{au}^{-1}$ to $0.4 \%{ }^{-1}$. And yet, observations showed that the jump in cosmic ray intensities took place over very short temporal and spatial scales (roughly a day and within fractions of an au), after which the cosmic ray intensities reached uniform, steady-state levels (where they have remained ever since). Models of radial behavior in $100 \mathrm{MeV}$ protons by $[13,14,15]$ demonstrated that small diffusion coefficients (within a factor 
of a few), could, indeed, cause heliospheric modulation to extend tens to a hundred au into the VLISM. However, reproducing the Voyager observations required a dramatic change in the diffusion coefficient between the heliosheath and VLISM. The model of [15] indicated that the perpendicular diffusion coefficient $\left(K_{\perp}\right)$ in the VLISM was reduced to $1 / 100$ of its heliospheric value and the parallel diffusion term $\left(K_{\|}\right)$was enhanced by over a factor of 100 . Meanwhile [13]'s best-matching simulation required that $K_{||} / K_{\perp}$ increase by a factor of $10^{10}$. Re-creating and understanding the the full implications of the cosmic ray behavior across the heliopause remains a challenge for most models, particularly owing to unknowns about the nature of the heliopause discontinuity, the small spatial resolution required, and the need to include accurate models of both the heliospheric and interstellar magnetic fields at either side of the boundary. As such, the topic remains an active area of research.

Meanwhile, [16] examined 3 to $346 \mathrm{MeV}$ cosmic ray protons measured by Voyager 1 over a roughly 2.5-year time period (from $\sim 2012.9$ to $\sim 2015.5$ ) and found no strong evidence of a radial intensity gradient, reporting a value consistent with zero, with a $1 \sigma$ upper limit of $0.06 \% \mathrm{au}^{-1}$. Prior to these observations, the nature of the gradient in the VLISM was also debated by a variety of authors, including several who expected a positive gradient that extended many au beyond the heliopause [17, 12, 18, 19] while others anticipated no gradient at all $[13,15,20,21,22]$. The abruptness of the heliopause transition, the negligible intensity gradient, and the remarkably similar energy spectra of cosmic rays measured by the two Voyager spacecraft provide strong evidence that the cosmic ray modulation boundary is likely only a fraction of an au from the heliopause, and that the energy spectra represents that of the unmodulated local interstellar medium.

\section{The Low-Energy Very Local Interstellar Spectra}

Several years after Voyager 1's heliopause crossing, [16] reported the long-awaited cosmicray measurement of the low-energy very local interstellar spectra. Below energies of several hundred $\mathrm{MeV} / \mathrm{nuc}$, observations of GCRs in the solar wind are completely inhibited by several factors, including adiabatic energy losses and the dominance of other particle populations (i.e., anomalous cosmic rays and solar energetic particles), leaving their spectra virtually unknown [23, $24,25]$. Voyager's arrival in the VLISM enabled the first measurements of $\mathrm{H}$ through Ni from as low as $3 \mathrm{MeV} /$ nuc up to several-hundred $\mathrm{MeV} /$ nuc, as well as electrons from 2.7 to $74 \mathrm{MeV}$. Figure 2 compares the energy spectra of electrons $\left(e^{+}+e^{-}\right), \mathrm{H}$, and He nuclei, measured by both Voyagers. The spectra are remarkably similar for the two spacecraft, despite their different measurement times (late-2012 vs. early 2019), large separation distance ( $167 \mathrm{au})$, and North- vs. South-ward ecliptic trajectories [26].

$\mathrm{H}$ and $\mathrm{He}$ show a broad maximum from $\sim 10$ to $50 \mathrm{MeV} /$ nuc that is preceded by a slight decrease at low energies and followed by a noticeable drop-off at $\mathrm{GeV}$ energies and higher. In general, their profiles are very similar, with a mostly energy-dependent intensity ratio $(\mathrm{H} / \mathrm{He} \simeq$ 12), which, as noted by [16], was not obvious at 1 au due to the effects of solar modulation. The electrons exhibit a power law (index of -1.3) that is consistent across the entire observed range. Also, the electron intensity below $\sim 50 \mathrm{MeV}$ dominates over $\mathrm{H}$ by about a factor of 50 , in contrast to the high energy trend observed at earth $(>10 \mathrm{GeV})$, for which the intensity ratio $(e / \mathrm{H})$ of electrons is only a few \% of $\mathrm{H}[16]$. 
In the recent years, these data, along with long-term observations at $1 \mathrm{AU}$ from missions like ACE, PAMELA and AMS-02 [27, 28, 29] have led to significant advancements in the modeling of solar modulation and cosmicray transport for particles of many different species $[30,31,32,33,34,34]$. The spectra also have important implications for astrophysics. For example, low-energy cosmic rays are thought to play a critical role in the chemistry and physics of molecular clouds by driving their ionization rates. However, [16] derived an ionization rate of 1.51 to $1.64 \times 10^{-17} \mathrm{~s}^{-1}$ - roughly 1 to 2 orders of magnitude lower than inferred by studies of diffuse molecular clouds [35] (see also review by [36]). [16] offered two plausible conjectures: (i) the low-energy cosmic ray spectra throughout the Galaxy could be spatially variable, or (ii) a suprathermal tail below Voyager's energy capabilities could significantly contribute to the ionization rate. However, evidence for the former is not well-supported by astro-chemical results, and the latter has since been ruled out by other studies [37]. Additional possibilities have also been explored, but so far the reason for the discrepancy remains unsolved. Knowledge of the low-energy cosmic ray energy spectra has contributed to a variety of other studies as well, including those which use of $\gamma$-rays to probe the $\mathrm{GeV}$ to $\mathrm{TeV}$ spectra at different locations in the galaxy $[38,39]$

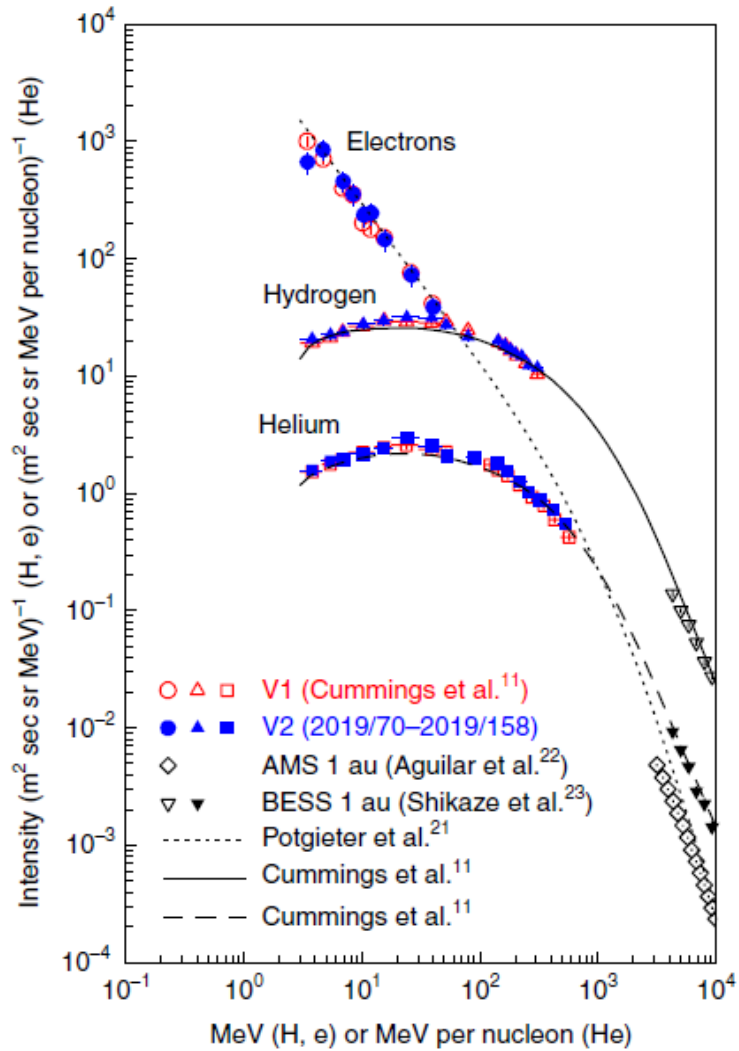

Figure 2. From [26]. Energy spectra of cosmic-ray H, He, and electrons $\left(\mathrm{e}^{+}+\mathrm{e}^{-}\right)$measured by Voyager $1(\mathrm{~V} 1$; red) and Voyager 2 (V2; blue) in the VLISM. Data shown were taken from 2012-342 to 2015-182 and 2019-70 to 2019-158 for Voyager 1 \& 2, respectively. Theoretical estimates of the interstellar spectra are also shown (dotted, solid, and dashed lines), along with high-energy observations from $1 \mathrm{au}$, for which modulation effects are likely small (black triangles and diamonds); see [16] for more details. and even to provide novel constraints for models of Dark Matter [40, 41, 42, 43].

\section{Time-Varying Pitch-Angle Dependent Cosmic Ray Anisotropies}

Shortly after Voyager 1 crossed the heliopause, the Low-Energy Charged Particle instrument (LECP) made an unanticipated discovery concerning the isotropy of cosmic rays. While intensities appeared mostly uniform and isotropic in the field-aligned $\left(0^{\circ}\right.$ pitch angle) and offpointing $\left(45^{\circ}\right.$ pitch angle) sectors of its rotating bi-directional telescope, proton-dominated measurements of cosmic rays from the $90^{\circ}$ sector revealed a pronounced, but smoothly-varying reduction in cosmic ray intensity [9]. 
[44] used proton-dominated rates ( $\gtrsim 20$ and $\gtrsim 70 \mathrm{MeV}$; median energies of $\sim 500 \mathrm{MeV}$ )

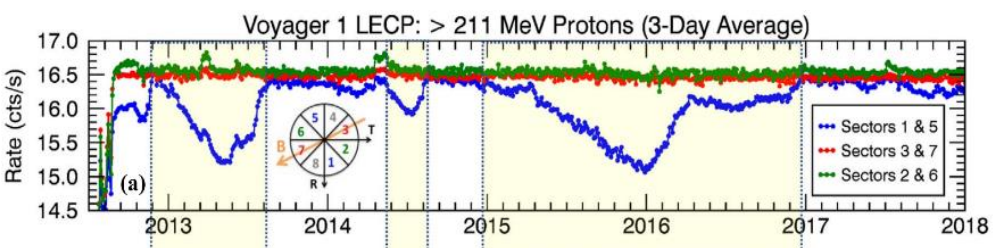
from Voyager 1's Cosmic Ray Subsystem (CRS) to analyze three distinct periods of anisotropy, observed from the time of the heliopause crossing (2012.65) up to 2018.0, as

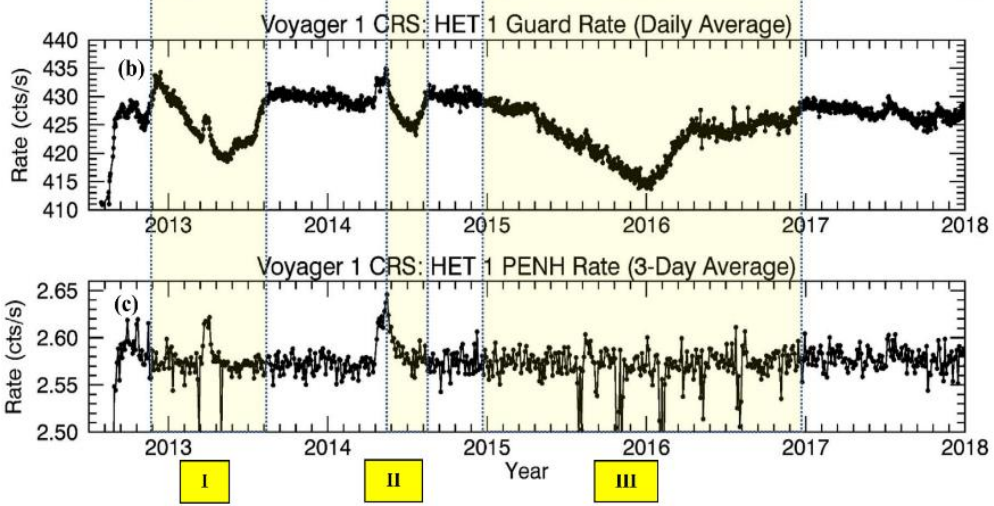
shown in Figure 3. These episodes ranged in duration from $\sim 100$ to $\sim 630$ days, with a small, but statistically-significant reduction of up to $3.8 \%$ in its omnidirectional counters. Viewing the anisotropy through CRS's body-fixed high-energy telescopes (HETs 1 \& 2)

Figure 3. From [44]. Cosmic ray counting rates in the VLISM, as viewed by Voyager's Low Energy Charged Particle (LECP) experiment and its Cosmic Ray Subsystem (CRS). During the roughly 6-year time period shown, three large anisotropy episodes occured (shaded in yellow), lasting 265 (I), 100 (II), and 630 (III) days. Intensity enhancements are also present, indicative of remote connections to several solartransient-induced shocks. (a) LECP's $>211 \mathrm{MeV}$ proton channel reveals the events' directionally-dependent nature, as they are only evident when its telescope is oriented approximately perpendicular to the magnetic field (e.g., circular diagram, Sectors 1 and 5). (b) High-resolution time profiles of the anisotropy events are also viewed by CRS's omnidirectional anticoincidence counters ( $\gtrsim 20 \mathrm{MeV}$; proton-dominated), but for these, the pitch-angle information is absent. (c) Although the CRS telescopes are body-fixed and do not typically view the anisotropy (i.e., the HET 1 PENH rate shown here; $\gtrsim 70 \mathrm{MeV}$; proton-dominated), occasional spacecraft pointing maneuvers cause the telescope's field of view to temporarily overlap with the $90^{\circ}$ pitch angle sector, as evidenced by the periodic dips.

proved a challenge, because the usual spacecraft pointing orientation placed the telescope field of views well outside of the $90^{\circ}$ pitch-angle sector. Nonetheless, by taking advantage of regular spacecraft rolls and offsetpointing maneuvers ${ }^{3}$, [44] confirmed that the dropouts were centered on $90^{\circ}$ pitch angle (to within $8.6^{\circ}$ ), and further used the off-pointing between the two telescopes to derive a characteristic width of the distribution. They found the anisotropy episodes to be well-described by a "notch" of missing particles in an otherwise uniform pitch angle distribution that was, on average, $22^{\circ}$ wide and $15 \%$ deep - thus signifying a broad and shallow depletion region.

\footnotetext{
${ }^{3}$ Multiple days of repointing that occured roughly every other month, which positioned telecope boresights to overlap with the $90^{\circ}$ pitch-angle viewing direction.
} 


\subsection{Anisotropy Events: A Plausible Physical Mechanism}

As for the mechanism behind the unusual pitch-angle-dependent behavior, one of the leading explanations was proposed by [45], who suggested the anisotropy events could arise due to trapping and cooling of energetic particles downstream of weak shocks. Several shocks had been observed by the Magnetometer [46] and were known instigators of the electron plasma oscillations measured by the Plasma Wave Subsystem (PWS) [47, 6]. Numerical simulations by [48] showed that a gradual compression, followed by a slow weakening of the magnetic field, could account for the pitch angle and time profiles of two types of interactions observed near the shocks: (i) short-lived cosmic-ray intensity enhancements reflected and accelerated prior to the shock's arrival and (ii) the long-duration anisotropic decreases arising from particles trapped and cooled downstream (ref. Figure 3). The results of [48]'s adiabatic heating and cooling model applied to a simple parabolic shock are shown in Figure 4.

As discussed by [44], the first anistropy event detected by Voyager 1 in 2013 provided
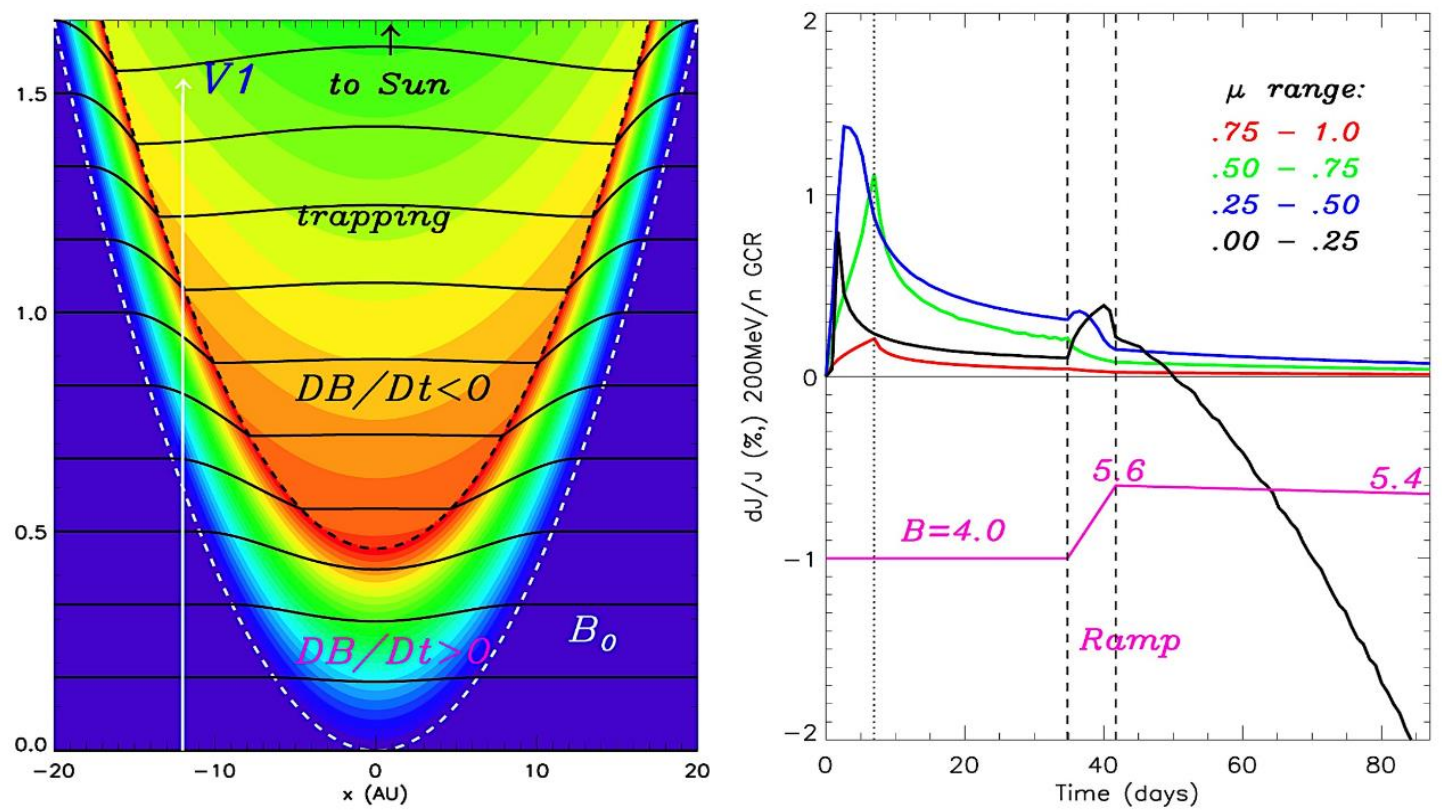

Figure 4. From [48]. Left Panel: Simulated behavior of magnetic structure within a shock moving away from the Sun at a speed of $40 \mathrm{~km} \mathrm{~s}^{-1}$. As Voyager 1 (V1) crosses through the field line system, it encounters two regions, the first comprised of the gradual compression of the shock itself (between the two dashed lines; DB/Dt $>0$ ), from which cosmic rays are accelerated and reflected producing short-lived intensity enhancements. The second region is characterized by gradual expansion $(\mathrm{DB} / \mathrm{Dt}<0)$ in which particles with the largest pitch angles (e.g., near $90^{\circ}$ ) are the most effectively trapped and cooled (strong fields shown in red, weak fields shown in blue). Right Panel: results from a simulation of $200 \mathrm{MeV}$ cosmic rays interacting with a simple compression (spherical shell) for an assumed magnetic field profile, B, that is gradually increasing over time (magenta). The various colors represent cosmic ray intensity responses for 4 different segments of pitch-angle, $\alpha$, where $\mu=\cos \alpha$. The intensity reduction is evident for pitch angles of $75^{\circ}$ to $90^{\circ}(\mu=0.00$ to 0.25$)$, in agreement with Voyager obserations.

compelling evidence concerning the influence of adiabatic cooling in the anisotropy's development. As shown in Figure 5, the field weakened as the particle intensities declined (i.e., from $\sim 2012.9$ to 2013.35; up to the anisotropy's minimum) and its recovery began as the field had stabilized around $\sim 0.46 \mathrm{nT}$ and was no longer expanding (Figure 5, 2013.35 to 2013.6). The third episode in 2015 showed similar, but not identical behavior in that its development occured in a two-step process (from $\sim 2014.65$ to $\sim 2015.35$ and 2015.35 to $\sim 2016.0$ ) as the field also weakened in two phases. 

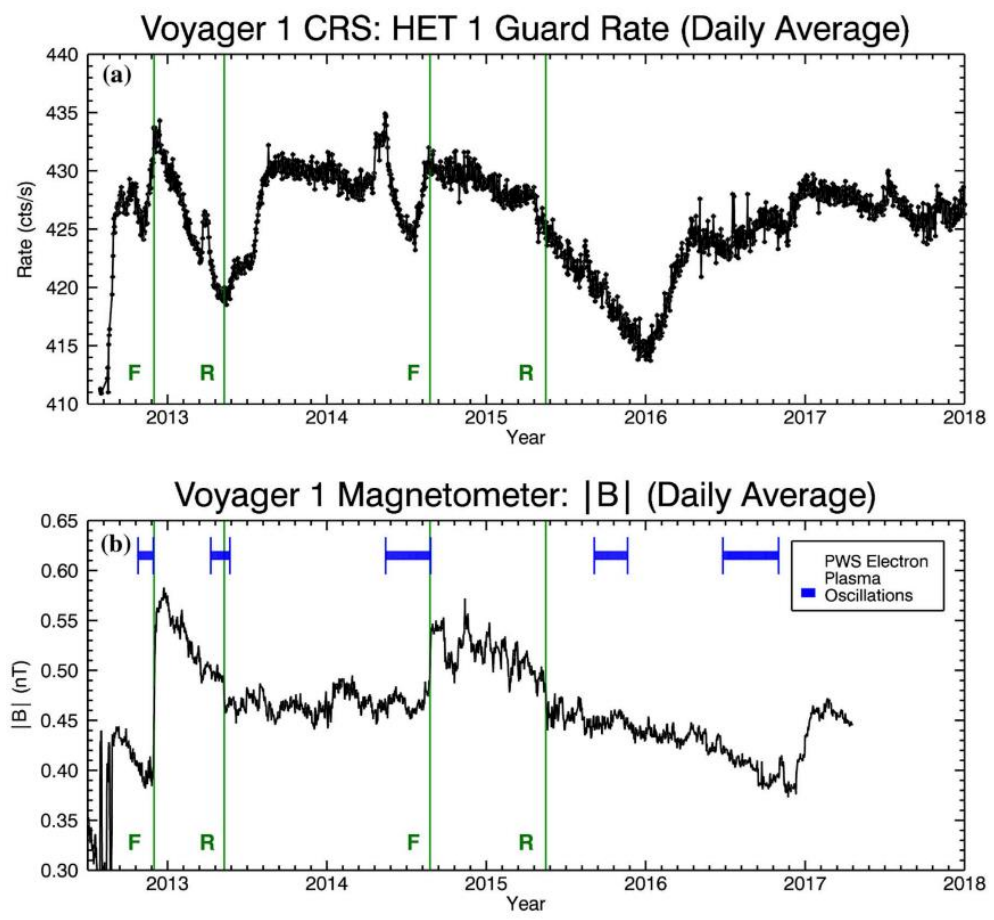

Figure 5. From [44]. Time profiles of cosmic rays (top panel; $\gtrsim 20 \mathrm{MeV}$; protondominated) and the magnetic field (bottom panel), in response to solar-caused disturbances in the VLISM. The timing of the electron plasma oscillations is captured by the horizontal blue bars, and the onset times of forward (F), and possible reverse $(\mathrm{R})$ shocks are delineated in green.
However, the data also showed clear deviations from the proposed adiabatic cooling/trapping mechanism. For example, the 2014 episode was not preceded by an obvious distrubance, and occured when the local fields were neither expanded nor compressed. Morevover, the 2015 episode recovered despite a continued drop in the local magnetic field. To address these inconsistencies, [44] considered several scenarios for which the trapping and cooling might occur (both insitu and remotely), eventually concluding that the anisotropy forms in a region which, "in addition to being affected by temporarily compressed fields from traveling disturbances, could also be affected by the presence of a steady state enhanced magnetic field near the heliopause". This steady-state field is consistent with the compressed field observed by IBEX at the heliopause near the ribbon [49, 50]. [51] similarly argued that the draping of the magnetic fields around the heliopause could be essential to the anisotropy's formation. However, they came to a different conclusion about where the trapping might occur: "the anisotropy-causing physical process that suppresses intensities at $\sim 90^{\circ}$ pitch angles relies on [cosmic rays] escaping from a single compression in the draping region, not on [cosmic rays] trapped between two compressions." As a whole, the mechanism introduced by $[48,45]$ is somewhat supported by observations, but there are many aspects of the events that are not yet fully understood.

\subsection{Anisotropy Events: Evidence of Species Dependence}

In a follow-on study, [52] expanded their approach to include electrons, along with protons in a few discrete low-energy bands. Their observations are summarized in Figure 6. In this, they reported an unexpected new finding: electrons exhibited no strong evidence of anisotropy. Unlike 
their proton counterparts, even electrons with near- $90^{\circ}$ pitch angles appeared mostly isotropic and unchanging (energies ranging from $\sim 3$ to $\sim 105 \mathrm{MeV}$ ). After ruling out pointing direction and energy dependence as possible explanations, [52] went on to explore five other possibilities to account for the lack of electron anisotropy: inneffective trapping, inneffective cooling, (iii) drifts, (iv) turbulence-induced scattering, and (v) alternative sources of scattering.

The first two possibilities addressed the anisotropy mechanism itself; could it be that electrons were less effectively trapped or cooled downstream of the interstellar shocks? The authors concluded that ineffective trapping was not the case for several reasons, including the fact that precursor "shock spike" intensity enhancement features (known
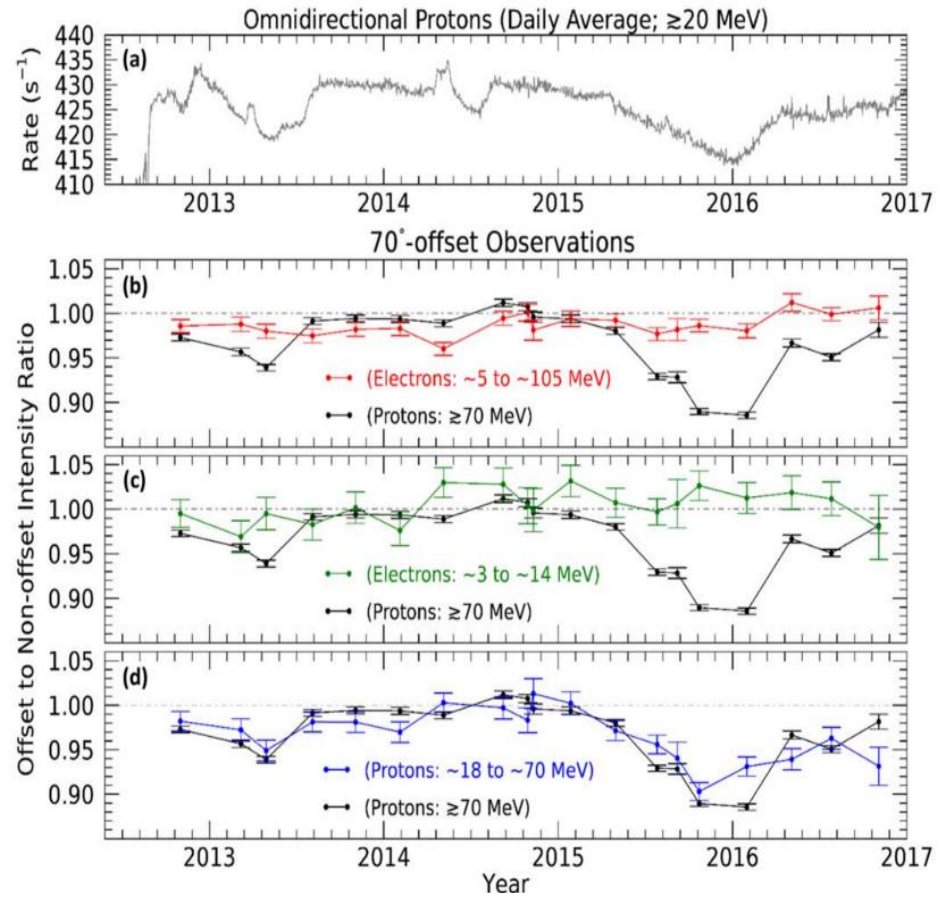

Figure 6. From [52]. Observations from the Voyager 1 Cosmic Ray Subsystem (CRS) reveal a lack of noticeable anisotropy in cosmic-ray electrons. Clear intensity reductions occur in omnidirectional protons $>20 \mathrm{MeV}$ (omnidirectional protons; panel a; grey) and bi-directional $>70 \mathrm{MeV}$ protons when the telescopes are re-pointed during $70^{\circ}$-offset maneuvers (panels b-d; black). $\sim 5$ to $\sim 105 \mathrm{MeV}$ electrons on a separate telescope show very little response, despite a being more directly aligned to view pitch angles of $90^{\circ}$ (panel b; red). Even electrons ( $\sim 3$ to $\sim 14 \mathrm{MeV}$; green) and protons $(\sim 18$ to $\sim 70 \mathrm{MeV}$; blue) on the same telescope differ in their responses (panels c \& d), eliminating possible differences due to pointing. The protons in panel $\mathrm{c}$ (blue) and electrons in panel $\mathrm{b}$ also cover similar energy ranges, and so the behavior likewise cannot be simply attributed to energy dependence. to precede the electron plasma oscillations) [47] were evident in both electrons and protons, indicating that both species were clearly interacting with the shock boundaries. Inneffective cooling was also ruled out, since the steeper spectrum [16] should yield more effective adiabatic cooling, and therefore produce a greater intensity change for electrons compared to protons - contrary to what the observations showed. Drifts were considered as another viable mechanism, particularly since they are known to influence the paths of particle propagation in a charge-dependent way. However, since curvature-gradient drifts (which dominate in the VLISM) have no divergence, they were not expected to directly contribute to adiabatic cooling or energy loss [53], thereby making it unclear as to what influence drifts may have (if any) on the anistropy events.

The role that turbulence might play in the development of these events (or lack thereof) proved perhaps the most puzzling. It seemed plausible to [52] that turbulence could scatter the electrons and thereby effectively erase evidence of their pitch angle distributions. However, the negatively-sloped power spectra reported by $[54,55]$ and $[56,57]$ suggested that amplitudes at resonantly-interactive wavelengths were 2-3 orders of magnitude larger for the lowest-energy protons compared to the highest energy used by [52]. Evidently, it was the protons, not the electrons, that should have been more effectively scattered by ambient turbulent fluctuations in the VLISM. However, as the authors noted, turbulence could instead participate in the formation of the anisotropies in protons or possibly contribute to the scattering of electrons in some other way. For example, an isotropic turbulence model by [58] demonstrated that pre-existing VLISM turbulence could effectively trap suprathermal protons (of a few $\mathrm{keV}$ in energy) with near- $90^{\circ}$ pitch angles through magnetic mirroring, and their results were generally consistent with the enhanced energetic neutral atom signature of the IBEX ribbon [59]. Although the Voyager 
anisotropies occur at much higher energies (few to hundred MeV's) and reflect energy losses rather than intensity gains, the role of turbulence in the pitch-angle-dependent perturbation of cosmic ray protons merits further exploration.

Returning to the possiblity of turbulence-induced scattering of electrons, several authors have found that the shocks which typically precede the anisotropy events likely change the local turbulence conditions $[60,55]$. For example, [61] performed a multi-scale analysis of turbulence in the VLISM using high-resolution data (48 s cadence) on Voyager 1. They found significant large-scale fluctuations, shock-induced turbulence, and intermittency on small scales, as well as magnetic energy flux significantly larger than reported by prior studies of turbulence in the VLISM [57]. The latter led the authors to suggest PUI instability as a candidate process for generating tubrulence in the high-frequency range, a regime that could potentially isotropize $\sim 1$ to $100 \mathrm{MeV}$ electrons. This topic, too, is recommended for follow-on study.

Lastly, [52] ventured beyond turbulence to suggest that the type of mechanism needed to explain their observations would most likely (i) depend on mass or charge, (ii) enable scattering through $90^{\circ}$ pitch angle near the resonant gap, and (iii) as a result of effective pitch-angle scattering, increase the probability for electrons to escape the magnetic trap and thereby prevent effective cooling. They further argued that electric fields - particularly electromagnetic ion cyclotron waves - could fulfill many of these conditions.

So far, the lack of anisotropy in cosmic-ray electrons still remains open question, and although several compelling options have been proposed, other reasonable mechanisms could also exist. The authors encourage the theoretical and modeling communities in particular to "push deeper into the explanation of these surprising and therefore fundamentally important observations."

\section{Transient Disturbances from the Heliosheath to the VLISM}

In addition to the multiple episodes of anisotropy in cosmic-ray protons, several other types of transient events have been regularly observed by both Voyager spacecraft in the VLISM. $[4,56]$ reported several weak laminar, quasi-perpendicular, subcritical shocks - a few thousand times thicker than their 1-au counterparts - as well as an unusual disturbance which comprised of $\mathrm{a} \sim 35$ day increase in the magnetic fields, followed by a $\sim 340$-day decay [5]. The latter was likely a time-delayed manifestation of the large increase in solar wind dynamic pressure responsible for inflating the heliosphere that mwas detected by the Interstellar Boundary Explorer beginning in

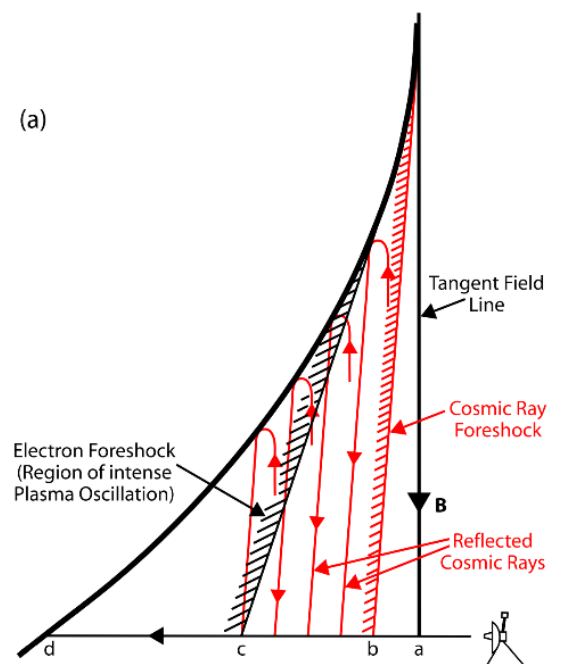

(b)

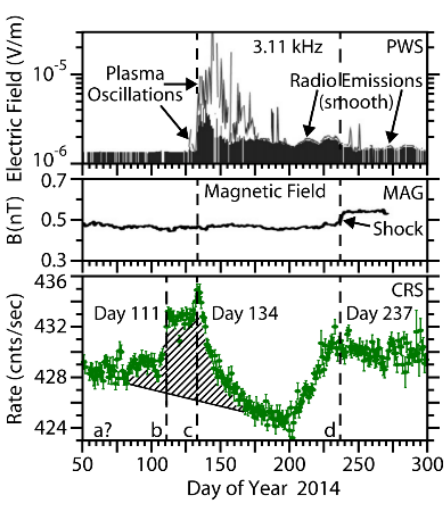

Figure 7. From [47]. (a) Foreshock schematic, illustrating precursor features encountered by Voyager before it crosses a shock. As the spacecraft initially crosses field lines connected to the shock, cosmic rays reflected and accelerated at the boundary should arrive first (red lines), followed by the electron plasma oscillations in the electron foreshock region (black hatching) and, sometimes (not always), the shock itself. (b) Electric field, magnetic field, and cosmic ray intensities measured during the 2014 event (recall Figure 3), with points "a", "b," "c," and "d" corresponding to the locations denoted in (a). late 2017 [62, 63, 64]. $[6,47,65]$ compared the locally-generated plasma emissions seen by PWS to those of short-lived ( 25-days) cosmic-ray intensity enhancements ("shock spikes") seen by CRS and LECP. Several of these events were found to accompany the weak quasiperpendicular shocks in the magnetic field, so $[47,65]$ formulated a model to describe the relationship of these collective measurements, known as the "foreshock 
model". According to this model, the cosmic ray and plasma events could be explained as shock precursors, analogous to those typically observed upstream of planetary bowshocks. The arrival time of each event was determined by the spacecraft's connectivity to the shock front, with the highest-energy particles reflected and accelerated from the shock first (e.g cosmic-ray electrons and protons in the comic ray foreshock region), followed by the electrons plasma oscillations (few to hundred $\mathrm{eV}$ ), and then, in many cases, the shock itself, as summarized by the schematic in Figure 7.

[65] tested the theory by using the timing of precursor enhancements in relativistic-energy cosmic ray electrons ( $\sim 5$ to $100 \mathrm{MeV})$ in conjuction with the onset times of the plasma oscillation events to estimate, for the first time, the energies of the electron beams responsible for the plasma oscillations. The values ( 20 to $100 \mathrm{eV}$; with an average of $\sim 50 \mathrm{eV}$ ) were found to be comparable to those responsible for driving Type-II radio bursts in the solar wind.

The influence of solar transients on the VLISM had long ago been anticipated by [66] who observed radio waves early on in the Voyager mission and after each successive solar maxima. The first two events were particularly dramatic in that they took place $\sim 400$ days after some of the largest Forbush decreases ever observed (1983-1984 and 1992-1994). [66] conjectured that the large solar transient events had coalesced to form merged interaction regions (MIRs), that survived out to the heliopause and drove shocks in the surrounding interstellar material, therby

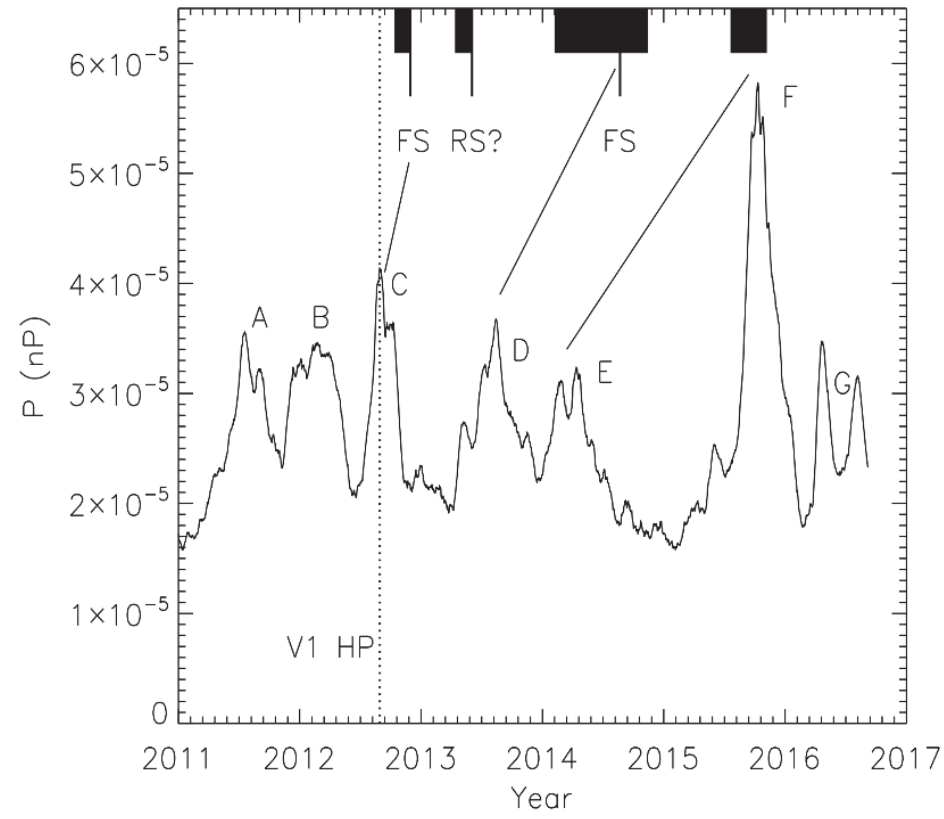

Figure 8. From [67]. Dynamic pressure peaks measured by the Voyager 2 plasma instrument in the heliosheath. The thick black lines at the top depict the timing and duration of the plasma oscillations, while the thin black lines indicate possible forward (FS) and reverse (RS) shocks observed by Voyager 1 in the VLISM. The dashed black line shows when Voyager 1 crossed the heliopause and the solid diagonal lines link disturbances at Voyager 2 that are the likely candidates for driving the plasma wave events at Voyager 1. producing the electron beams responsible for generating plasma emissions.

A prime opportunity to gain more insight into these occurrences arose during the $\sim 6$ years when Voyager 2 was in the heliosheath while Voyager 1 was in the VLISM. This was pursued by [67]. Noting that the arrival of solar maximum in the heliosheath appeared in mid-2012, they identified 5 possible MIRs observed by Voyager 2 (from 2012.5 to 2016.5) as candidates for disturbances that could eventually seen by Voyager 1 , shown in Figure 8. The events in the heliosheath were characterized by large increases in plasma pressure (enhancements of $\sim 50 \%$ to $300 \%$ ) followed by pronounced decreases in

cosmic ray intensity (the magnetic field data were not available during this time). The largest of these pressure pulses (observed by Voyager 2 in late 2015) has since been associated with the previously-mentioned pressure front seen by IBEX (enhanced ENA emissions at 1 au in late 2017; [63] \& references therein) and Voyager 1 (e.g the unusual, long-duration magnetic disturbance from late 2016 to late 2017) [5]. By comparing arrival times of MIRs in the heliosheath to the timing of plasma oscillations and shocks in the VLISM, [67] concluded: "the data seem consistent with the hypothesis that the pressure pulses observed at [Voyager 2] are driving the transients observed in the LISM by [Voyager 1]." 
[68] found further evidence in the cosmic rays to support [67]'s claims. They discovered cosmic ray disturbances at each spacecraft which showed remarkable similarities (having a correlation of $91.2 \%$ ), indicating that these events were very likely linked by a common cause. Evidently, a global merged interaction region (GMIR), had passed through the heliosheath in mid-2012, and after its detection by Voyager 2, collided with the heliopause, transmitted a pressure wave into the VLISM, and drove the first large anisotropy event seen by Voyager 1 just 130 days later. Using the observed time delay, they calculated the average speed of the GMIR through the heliosheath as a function of temperature in the VLISM. The speed of the event, along with in-situ measurements of the magnetic field and plasma and several wellsupported assumptions enabled them to derive previously unmeasured properties of the heliosheath - a range of plausible sound speeds and total effective pressures.

The results were obtained for a range of VLISM temperatures ${ }^{4}$, but [68] exemplified their findings using a nominal temperature of 20,000 K (illustrated in Figure 9), consistent with expectations from models [69]. However, results from Voyager 2 have more recently

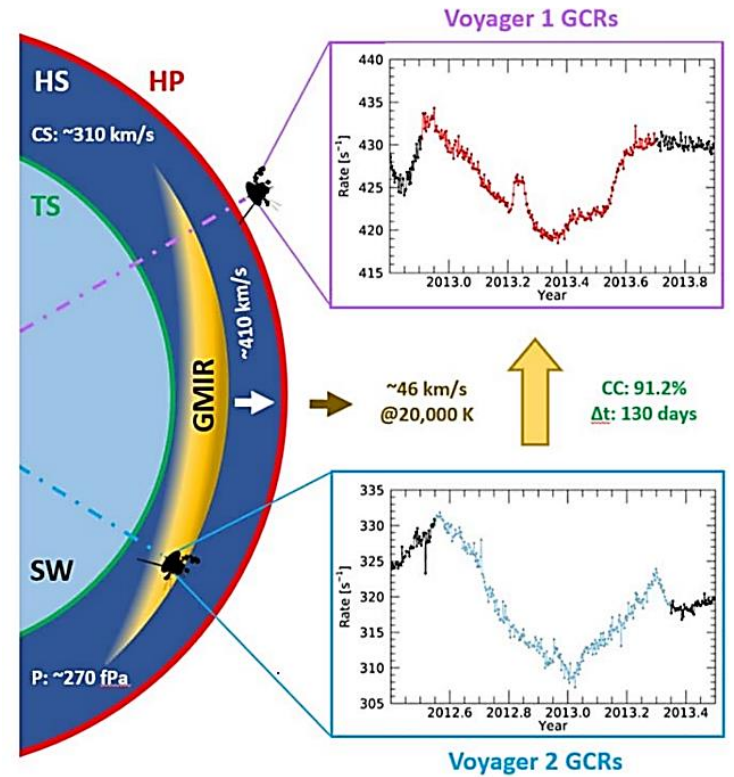

Figure 9. From [68]. Illustration of a GMIR that likely produced an isotropic disturbance of cosmic rays at Voyager 2, collided with the heliopause, and transmitted a pressure wave into the VLISM, thereby producing a causally related, but anisotropic disturbance at Voyager 1. The respective cosmic ray observations shown in red (Voyager 1) and blue (Voyager 2) occurred 130 days apart, with a high coefficient of correlation $(91.2 \%)$. The heliosheath pressure $(\mathrm{P})$ and sound speed (CS) listed here were calculated assuming a VLISM temperature of $20,000 \mathrm{~K}$. revealed VLISM temperatures that are somewhat higher than anticipated $(30,000 \mathrm{~K}$ to $50,000 \mathrm{~K})$ [8], so it is the $40,000 \mathrm{~K}$ results that we summarize here (ref. Tables $1 \& 2$ of [68]). For $\mathrm{T}_{\mathrm{VLSIM}}=$ $40,000 \mathrm{~K}$, [68] calculated speeds of $v_{H S}=392 \pm 40 \mathrm{~km} \mathrm{~s}^{-1}$ for the GMIR in the heliosheath and $v_{V L I S M}=51.6 \pm 4.9 \mathrm{~km} \mathrm{~s}^{-1}$ for the pressure pulse in the VLISM. From this, they derived an average sound speed of $C_{H S}=299 \pm 31 \mathrm{~km} \mathrm{~s}^{-1}$ and total effective pressure of $P_{\text {total }}=$ $242 \pm 50 \mathrm{fPa}$ in the heliosheath. It is interesting to compare this latter result to partial pressures inferred from IBEX and Voyager observations (see [68], Table 1). Constituents of this total pressure are as follows: $1.3 \%=$ thermal; $2.2 \%=$ magnetic; $\sim 12 \%=$ dynamic pressure of the solar wind; $\sim 21 \%=$ anomalous and galactic cosmic rays; $\sim 45 \%=$ pickup ions. This leaves $18.5 \%$ that is presently unaccounted for (e.g., not directly measured by Voyager or IBEX), possibly due to electrons [70, 71].

The findings of [68] are supported by more recent studies, such as [72, 73], but also posed a challenging question: given their surprisingly similar time profiles, why was the cosmic ray disturbance isotropic in the heliosheath but highly anisotropic in the VLISM? An interesting solution was put forth by [74], who simulated the perturbation of cosmic-rays by GMIRs in the heliosheath and employed a Vlasov-Fokker-Planck equation to allow for the transport of particles having significant anisotropy. Their model demonstrated that the Forbush-decrease-like modulation of cosmic rays by GMIRs in the heliosheath vs. the anisotropic, pitch-angle limited modulation of cosmic rays in the VLISM could be accounted for by the turbulence in the two regimes. Due to the weak scattering of the VLISM, the memories of pitch angles are preserved as particles are trapped and cooled in regions of rarefied magnetic field downstream of interstellar shocks. In the heliosheath, similar adiabatic cooling likely takes place, but the strongly turbulent magnetic field rapidly scatters particles at all pitch angles, forming a mostly isotropic distribution.

\footnotetext{
${ }^{4}$ Temperatures ranging from 7,500 to $40,00 \mathrm{~K}$ led to pressure estimates from $292 \pm 60$ to $252 \pm 50 \mathrm{fPa}$.
} 


\section{Conclusion}

The nearly decade of in-situ measurements in the VLISM by the two Voyager spacecraft has resulted in a rich new data set that continues to yield fresh insights and exciting discoveries, particularly for cosmic-rays. The work we have highlighted here is mostly derived from alreadypublished findings from Voyager 1, but each type of observation mentioned has a Voyager 2 counterpart that is soon to be analyzed and deciphered. Over the past 10 years, substantial progress has been made concerning the fundamental physics of cosmic rays and also towards broader questions in heliophysics. By sampling this new plasma environment, we have probed the global properties of the solar wind, measured how the interstellar medium influences our heliosphere, and witnessed how the Sun and heliosphere interact with their surroundings. Indeed, these represent historic achievements on behalf of all humankind as we finally explore our stellar neighborhood from the outside-in, rather than inside-out. But there is also still more work to be done and we look forward to future studies that will undoubtedly address open questions, further current understanding, and help us gain new wisdom about our place within the galaxy.

\section{References}

[1] S. M. Krimigis, R. B. Decker, E. C. Roelof, et al., "Search for the Exit: Voyager 1 at Heliosphereltextquoterights Border with the Galaxy," Science, vol. 341, pp. 144-147, 2013.

[2] E. C. Stone, A. C. Cummings, F. B. McDonald, B. C. Heikkila, N. Lal \& W. R. Webber, "Voyager 1 Observes Low-Energy Galactic Cosmic Rays in a Region Depleted of Heliospheric Ions," Science, vol. 341, pp. 150-153, 2013.

[3] W. R. Webber \& F. B. McDonald, "Recent Voyager 1 data indicate that on 25 August 2012 at a distance of 121.7 AU from the Sun, sudden and unprecedented intensity changes were observed in anomalous and galactic cosmic rays," Geophysical Research Letters, vol. 40, pp. 1665-1668, 2013.

[4] L. F. Burlaga, N. F. Ness \& E. C. Stone, "Magnetic Field Observations as Voyager 1 Entered the Heliosheath Depletion Region," Science, vol. 341, pp. 147-150, 2013.

[5] L. F. Burlaga, N. F. Ness, D. B. Berdichevsky, et al., "Magnetic field and particle measurements made by Voyager 2 at and near the heliopause," Nature Astronomy, vol. 3, pp. 1007-1012, 2019.

[6] D. A. Gurnett, W. S. Kurth, L. F. Burlaga \& N. F. Ness, "In Situ Observations of Interstellar Plasma with Voyager 1," Science, vol. 341, pp. 1489-1492, 2013.

[7] D. A. Gurnett \& W. S. Kurth, "Plasma densities near and beyond the heliopause from the Voyager 1 and 2 plasma wave instruments," Nature Astronomy, vol. 3, pp. 1024-1028, 2019.

[8] J. D. Richardson, J. W. Belcher, P. Garcia-Galindo \& L. F. Burlaga, "Voyager 2 plasma observations of the heliopause and interstellar medium," Nature Astronomy, vol. 3, pp. 1019-1023, 2019.

[9] S. M. Krimigis, R. B. Decker, E. C. Roelof, et al., "Energetic charged particle measurements from Voyager 2 at the heliopause and beyond," Nature Astronomy, vol. 3, pp. 997-1006, 2019.

[10] V. Florinski, J. R. Jokipii, F. Alouani-Bibi \& J. A. le Roux, "Energetic Particle Anisotropies at the Heliospheric Boundary," The Astrophysical Journal, vol. 776, p. L37, 2013.

[11] V. Florinski, E. C. Stone, A. C. Cummings \& J. A. le Roux, "Energetic Particle Anisotropies at the Heliospheric Boundary. II. Transient Features and Rigidity Dependence," The Astrophysical Journal, vol. 803, p. 47, 2015.

[12] R. D. Strauss \& M. S. Potgieter, "Where does the heliospheric modulation of galactic cosmic rays start?" Advances in Space Research, vol. 53, pp. 1015-1023, 2014.

[13] X. Luo, M. Zhang, M. Potgieter, X. Feng \& N. V. Pogorelov, "A Numerical Simulation of CosmicRay Modulation Near the Heliopause," The Astrophysical Journal, vol. 808, p. 82, 2015.

[14] X. Luo, M. S. Potgieter, M. Zhang, et al., "A Numerical Simulation of Cosmic Ray Modulation Near the Heliopause. II. Some Physical Insights," The Astrophysical Journal, vol. 826, p. 182, 2016.

[15] M. Zhang, X. Luo \& N. Pogorelov, "Where is the cosmic-ray modulation boundary of the heliosphere?" Physics of Plasmas, vol. 22, p. 091501, 2015. 
[16] A. C. Cummings, E. C. Stone, B. C. Heikkila, N. Lal, et al., "Galactic Cosmic Rays in the Local Interstellar Medium: Voyager Observations and Model Results," The Astrophysical Journal, vol. 831 , p. 18, October 2016.

[17] R. D. Strauss, M. S. Potgieter, S. E. S. Ferreira, H. Fichtner \& K. Scherer, "Cosmic Ray Modulation Beyond the Heliopause: A Hybrid Modeling Approach," The Astrophysical Journal, vol. 765, p. L18, February 2013.

[18] K. Herbst, B. Heber, A. Kopp, O. Sternal, \& F. Steinhilber, "The Local Interstellar Spectrum beyond the Heliopause: What can be Learned from Voyager in the Inner Heliosheath?" The Astrophysical Journal, vol. 761, n. 1, p. 17, 2012.

[19] K. Scherer, H. Fichtner, R. D. Strauss, S. E. S. Ferreira, M. S. Potgieter \& H. J. Fahr, "On Cosmic Ray Modulation beyond the Heliopause: Where is the Modulation Boundary?" The Astrophysical Journal, vol. 735, n. 2, p. 128, 2011.

[20] X. Guo \& V. Florinski, "Galactic Cosmic-Ray Modulation Near the Heliopause," The Astrophysical Journal, vol. 793, p. 18, 2014.

[21] J. R. Jokipii, "Cosmic rays in the outer heliosphere and nearby interstellar medium," in The Outer Heliosphere: The Next Frontiers, Amsterdam, Pergamon Press, 2001, p. 513.

[22] J. Kota \& J. R. Jokipii, "Are Cosmic Rays Modulated Beyond the Heliopause?" The Astrophysical Journal, vol. 782, p. 24, 2014.

[23] M. S. Potgieter, "Solar Modulation of Cosmic Rays," Living Reviews in Solar Physics, vol. 10, p. 3 , June 2013.

[24] M. E. Wiedenbeck, "Cosmic-Ray Energy Spectra and Time Variations in the Local Interstellar Medium: Constraints and Uncertainties," Space Science Reviews, vol. 176, n. 1-4, pp. 35-46, 2013.

[25] R. D. Strauss, M. S. Potgieter, A. Kopp, \& I. Busching, "On the propagation times and energy losses of cosmic rays in the heliosphere," Journal of Geophysical Research, vol. 116, n. A12, p $105,2011$.

[26] E. C. Stone, A. C. Cummings, B. C. Heikkila, \& N. Lal, "Cosmic ray measurements from Voyager 2 as it crossed into interstellar space," Nature Astronomy, vol. 3, pp. 1013-1018, 2019.

[27] C.-R. Zhu, Q. Yuan \& D.-M. Wei, "Studies on Cosmic-Ray Nuclei with Voyager, ACE, and AMS02. I. Local Interstellar Spectra and Solar Modulation," The Astrophysical Journal, vol. 863, p. 119, 2018.

[28] A. M. Galper, R. Sparvoli, O. Adriani, et al., "The PAMELA experiment: a decade of Cosmic Ray Physics in space," Journal of Physics: Conference Series, vol. 798, p. 012033, 2017.

[29] C. Evoli, R. Aloisio \& P. Blasi, "Galactic cosmic rays after the AMS-02 observations," Physical Review D, vol. 99, p. 103023, 2019.

[30] D. Bisschoff, M. S. Potgieter \& O. P. M. Aslam, "New Very Local Interstellar Spectra for Electrons, Positrons, Protons, and Light Cosmic Ray Nuclei," The Astrophysical Journal, vol. 878, p. 59, 2019.

[31] Z.-N. Shen, G. Qin, P. Zuo \& F. Wei, "Modulation of Galactic Cosmic Rays from Helium to Nickel in the Inner Heliosphere," The Astrophysical Journal, vol. 887, p. 132, 2019.

[32] E. Fiandrini, N. Tomassetti, B. Bertucci, F. Donnini, M. Graziani, B. Khiali, \& A. Reina Conde, "Numerical modeling of cosmic rays in the heliosphere: Analysis of proton data from AMS-02 and PAMELA," Physical Review D, vol. 104, n. 2, p. 023012, 2021.

[33] M. Hanasz, A. W. Strong \& P. Girichidis, "Simulations of cosmic ray propagation," Living Reviews in Computational Astrophysics, vol. 7, p. 2, 2021.

[34] M. J. Boschini, S. Della Torre, M. Gervasi, et al., "Deciphering the Local Interstellar Spectra of Secondary Nuclei with the Galprop/Helmod Framework and a Hint for Primary Lithium in Cosmic Rays," The Astrophysical Journal, vol. 889, n. 2, 2020.

[35] N. Indriolo, D. A. Neufeld \& M. E. A. Gerin, "Herschel Survey of Galactic OH+, H2O+, and $\mathrm{H} 3 \mathrm{O}+$ : Probing the Molecular Hydrogen Fraction and Cosmic-Ray Ionization Rate," The Astrophysical Journal, vol. 800, n. 1, p. 40, 2015.

[36] M. Padovani, A. V. Ivlev, D. Galli, S. S. R. Offner, N. Indriolo, D. Rodgers-Lee, A. Marcowith, P. Girichidis, A. M. Bykov \& J. M. D. Kruijssen, "Impact of Low-Energy Cosmic Rays on Star Formation," Space Science Reviews, vol. 216, p. 29, 2020. 
[37] S. Recchia, V. H. M. Phan, S. Biswas \& S. Gabici, "Can a cosmic ray carrot explain the ionization level in diffuse molecular clouds?" Monthly Notices of the Royal Astronomical Society, vol. 485, pp. 2276-2280, 2019.

[38] A. Neronov, D. Malyshev \& D. V. Semikoz, "Cosmic-ray spectrum in the local Galaxy," Astronomy and Astrophysics, vol. 606, p. A22, 2017.

[39] D. A. Prokhorov \& S. Colafrancesco, "Testing spatial uniformity of the CR spectrum in the local ISM with gamma-ray observations," Monthly Notices of the Royal Astronomical Society, vol. 478, pp. 2939-2947, 2018.

[40] M. Boudaud, J. Lavalle \& P. Salati, "Novel Cosmic-Ray Electron and Positron Constraints on MeV Dark Matter Particles," Physical Review Letters, vol. 119, p. 021103, 2017.

[41] M. Boudaud \& M. Cirelli, "Voyager 1 e+/- Further Constrain Primordial Black Holes as Dark Matter," Physical Review Letters, vol. 122, p. 041104, 2019.

[42] M. Boudaud, T. Lacroix, M. Stref, \& J. Lavalle, "Robust cosmic-ray constraints on p-wave annihilating MeV dark matter," Physical Review D, vol. 99, p. 061302, 2019.

[43] H. Motz, H. A. Y. Okada \& K. Kohri, "Cosmic-ray signatures of dark matter from a flavor dependent gauge symmetry model with neutrino mass mechanism," Physical Review D, vol. 102, n. 8, p. 083019, 2020.

[44] J. S. Rankin, E. C. Stone, A. C. Cummings, D. J. McComas, N. Lal \& B. C. Heikkila, "Galactic Cosmic-Ray Anisotropies: Voyager 1 in the Local Interstellar Medium," The Astrophysical Journal, vol. 873, p. 46, 2019.

[45] J. R. Jokipii \& J. Kóta, "Interpretation of the Disturbance in Galactic Cosmic Rays Observed on Voyager 1 beyond the Heliopause," Astrophysical Journal Letters, vol. 794, p. L4, 2014.

[46] L. F. Burlaga \& N. F. Ness, "Observations of the Interstellar Magnetic Field in the Outer Heliosheath: Voyager 1," The Astrophysical Journal, vol. 829, p. 134, 2016.

[47] D. A. Gurnett, W. S. Kurth, E. C. Stone, A. C. Cummings, S. M. Krimigis, R. B. Decker, N. F. Ness \& L. F. Burlaga, "Precursors to Interstellar Shocks of Solar Origin," The Astrophysical Journal, vol. 809, p. $121,2015$.

[48] J. Kóta \& J. R. Jokipii, "Transient Cosmic-ray Events beyond the Heliopause: Interpreting Voyager1 Observations," The Astrophysical Journal, vol. 839, p. 126, 2017.

[49] D. J. McComas, F. Allegrini, P. Bochsler, Bzowski, et al., "Global Observations of the Interstellar Interaction from the Interstellar Boundary Explorer (IBEX)," Science, vol. 326, p. 959, 2009.

[50] E. J. Zirnstein, J. Heerikhuisen, H. O. Funsten, G. Livadiotis, D. J. McComas \& N. V. Pogorelov, "Local Interstellar Magnetic Field Determined from the Interstellar Boundary Explorer Ribbon," The Astrophysical Journal Letters, vol. 818, p. L18, 2016.

[51] M. E. Hill, R. C. Allen, P. Kollmann, L. E. Brown, et al., "Influence of Solar Disturbances on Galactic Cosmic Rays in the Solar Wind, Heliosheath, and Local Interstellar Medium: Advanced Composition Explorer, New Horizons, and Voyager Observations," The Astrophysical Journal, vol. 905, p. 69, 2020.

[52] J. S. Rankin, D. J. McComas \& N. A. Schwadron, "Galactic Cosmic-ray Anisotropies: Electrons Observed by Voyager 1 in the Very Local Interstellar Medium," The Astrophysical Journal, vol. 895 , p. 103, 2020.

[53] J. R. Jokipii, E. H. Levy \& W. B. Hubbard, "Effects of particle drift on cosmic-ray transport. I. General properties, application to solar modulation.," The Astrophysical Journal, vol. 213, pp. 861868, 1977.

[54] G. P. Zank, S. Du \& P. Hunana, "The Origin of Compressible Magnetic Turbulence in the Very Local Interstellar Medium," The Astrophysical Journal, vol. 842, p. 114, 2017.

[55] G. P. Zank, M. Nakanotani \& G. M. Webb, "Compressible and Incompressible Magnetic Turbulence Observed in the Very Local Interstellar Medium by Voyager 1," The Astrophysical Journal, vol. 887, p. 116, 2019.

[56] L. F. Burlaga, V. Florinski \& N. F. Ness, "In Situ Observations of Magnetic Turbulence in the Local Interstellar Medium," Astrophysical Journal Letters, vol. 804, p. L31, 2015.

[57] L. F. Burlaga, V. Florinski \& N. F. Ness, "Turbulence in the Outer Heliosheath," The Astrophysical Journal, vol. 854, p. 20, 2018. 
[58] J. Giacalone \& J. R. Jokipii, "A New Model for the Heliosphere IBEX Ribbon," Astrophysical Journal Letters, vol. 812, p. L9, 2015.

[59] E. J. Zirnstein, J. Giacalone, R. Kumar, D. J. McComas, M. A. Dayeh, \& J. Heerikhuisen, "Turbulence in the Local Interstellar Medium and the IBEX Ribbon," The Astrophysical Journal, vol. 888, p. 29, 2020.

[60] F. Fraternale, N. V. Pogorelov, J. D. Richardson \& D. Tordella, "Magnetic Turbulence Spectra and Intermittency in the Heliosheath and in the Local Interstellar Medium," The Astrophysical Journal, vol. 872, p. 40, 2019.

[61] F. Fraternale \& N. Pogorelov, "Waves and Turbulence in the Very Local Interstellar Medium: From Macroscales to Microscales," The Astrophysical Journal, vol. 906, n. 2, p. 75, 2021.

[62] D. J. McComas, M. A. Dayeh, H. O. Funsten, et al., "Heliosphere Responds to a Large Solar Wind Intensification: Decisive Observations from IBEX," Astrophysical Journal Letters, vol. 856, p. L10, 2018.

[63] D. J. McComas, M. Bzowski, M. A. Dayeh, et al., "Solar Cycle of Imaging the Global Heliosphere: Interstellar Boundary Explorer (IBEX) Observations from 2009-2019," The Astrophysical Journal Supplement Series, vol. 248, n. 2, p. 26, 2020.

[64] E. J. Zirnstein, J. Heerikhuisen, D. J. McComas, et al., "Simulation of the Solar Wind Dynamic Pressure Increase in 2014 and Its Effect on Energetic Neutral Atom Fluxes from the Heliosphere," The Astrophysical Journal, vol. 859, p. 104, 2018.

[65] D. A. Gurnett, W. S. Kurth, E. C. Stone, et al., "A Foreshock Model for Interstellar Shocks of Solar Origin: Voyager 1 and 2 Observations," The Astronomical Journal, vol. 161, p. 11, 2020.

[66] D. A. Gurnett, W. S. Kurth, S. C. Allendorf \& R. L. Poynter, "Radio Emission from the Heliopause Triggered by an Interplanetary Shock," Science, vol. 262, n. 5131, pp. 199-203, 1993.

[67] J. D. Richardson, C. Wang, Y. D. Liu, J. Šafránková, Z. Němeček \& W. S. Kurth, "Pressure Pulses at Voyager 2: Drivers of Interstellar Transients?" The Astrophysical Journal, vol. 834, p. 190, 2017.

[68] J. S. Rankin, D. J. McComas, J. D. Richardson, \& N. A. Schwadron, "Heliosheath Properties Measured from a Voyager 2 to Voyager 1 Transient," The Astrophysical Journal, vol. 883, p. 101, 2019.

[69] G. P. Zank, J. Heerikhuisen, N. V. Pogorelov, R. Burrows \& D. McComas, "Microstructure of the Heliospheric Termination Shock: Implications for Energetic Neutral Atom Observations," The Astrophysical Journal, vol. 708, n. 2, pp. 1092-1106, 2010.

[70] H. J. Fahr \& M. Heyl, "Probing the thermodynamic conditions of the heliosheath plasma by shock wave propagation," Astronomy \& Astrophysics, vol. 642, p. A144, 2020.

[71] H. J. Fahr \& M. Heyl, "Suprathermal plasma distribution functions with relativistic cut-offs,» Monthly Notices of the Royal Astronomical Society, vol. 491, n. 3, pp. 3967-3973, 2020.

[72] K. Dialynas, A. Galli, M. A. Dayeh, et al., "Combined $10 \mathrm{eV}$ to $344 \mathrm{MeV}$ Particle Spectra and Pressures in the Heliosheath along the Voyager 2 Trajectory," The Astrophysical Journal, vol. 905, p. L24, 2020.

[73] D. B. Reisenfeld, M. Bzowski, H. O. Funsten, et al., "A Three-dimensional Map of the Heliosphere from IBEX," The Astrophysical Journal Supplement Series, vol. 254, p. 40, 2021.

[74] M. Zhang \& N. Pogorelov, "Modulation of Galactic Cosmic Rays by Plasma Disturbances Propagating Through the Local Interstellar Medium in the Outer Heliosheath," The Astrophysical Journal, vol. 895, p. 1, 2020.

[75] M. S. Potgieter, "The global modulation of cosmic rays during a quiet heliosphere: A modeling perspective," Advances in Space Research, vol. 60, pp. 848-864, 2017.

[76] D. J. McComas, M. A. Dayeh, H. O. Funsten et al., "Heliosphere Responds to a Large Solar Wind Intensification: Decisive Observations from IBEX," The Astrophysical Journal, vol. 856, p. L10, 2018.

[77] G. P. Zank, "Faltering Steps Into the Galaxy: The Boundary Regions of the Heliosphere," Annual Review of Astronomy and Astrophysics, vol. 53, pp. 449-500, 2015. 